\title{
A Privacy - LeARning O BJECTS IDENTITY SySTEM FOR SMARTPHONES BASED ON A VIRTUAL LEARNING ENVIRONMENT DATABASE
}

\author{
LeThanhHieu*\#, JinHai ${ }^{*}$, DeQingZou ${ }^{*}$, WangDaiBin ${ }^{*}$ \\ *Cluster Grid Computing Laboratory (CGCL) \\ Computer Science Department, HuaZhong University of Science and Technology \\ Address 1037 Luoyu Road, Wuhan, China \\ \#Hue University's college of Education \\ Address: 32 Le Loi, Hue city, Viet Nam
}

\section{ABSTRACT}

Smartphones are widely used today, with many features such as GPS map navigation, capturing photos with camera equipment such as digital camera, internet connection via wifi or $3 G$ devices that function as computers. These devices are being used for various purposes including online learning, where learners can study from anywhere and anytime for example in the street, home, office and school. However, identifing a method by which teachers in these virtural environements can remember their learners "faces" in the classroom or manage "Identification Number Student" (ID student or user) is not reliable when the teacher cannot see all of the learners in the class or know who is online from a particular account. In this paper, we propose a system, Android Virtual Learner Identify (AVLI), which collects images captured by the face of the learning object directly from the camera, the location of the learner by identifing where the learner is studying and configuration of information including Time, Mac, IP addresses, IMEI number and location via GPS. The systen then saves learner profiles to help the teacher or education managers on the Virtual Learning Environment (VLE) identify learning object. We used the VLE that we built on mobile.ona.vn domain. We implemented the AVLI prototype Android phone with solution password encryption and images taken directly from the camera to ensure that the information is transmitted and stored securely in the Virtual Learning Environment System Database (VLE Data) of learning objects while preserving the ability to identify learning objects by a teacher or education manager.

In addition, we solve the problem of image size on a smartphone device by compressing images. We demonstrate our solution which is secure enough to prevent fraud of the learner as well as the transmission of data from the client device to the server with three layers of protection by taking images directly from the camera, converting images from string and encoding them before transmission.

\section{KEYWORDS}

Privaxy, security Mlearning, learning management system, Virtual Learning Environment System

\section{INTRODUCTION}

With the development of technology, smartphones have become increasingly popular. They offer "all in one," which goes beyond the features of traditional telephony. Its features include phone, calendar, games, and address management, where users can browse the web using internet connectivity through General Packet Radio Service (GPRS), Data network cards or connect phone service form (sim card) allows users to read email, view photos, and use video 
applications, such as satellite navigation, photography. According to Gartner: Worldwide mobile phone sales to end users totalled nearly 426 million units in the first quarter of 2013. In the Smartphone operating system (OS) market, Android continued to increase its lead, with nearly 50 percent more Android Smart phones in the market than a year ago. Mr. Anshul said "There are two clear leaders in the OS market and Android's dominance in the OS market is unshakable" [1]. Thus, smartphones have become an important role in our lives, including online learning activities.

In the process of online learning activities, identification of the learner objects, if only managed by teachers with students in the classroom as it is in the traditional education environment or managed via an student identification number, is not reliable when the teacher cannot remember all of their learners in the class or know who is online with a particular account, even though it may have a password, learners can send photos or coursework and there it is difficult for the teacher to know whether or not the content has been edited before. The solution requires to identify who the learner is with the combination multiple forms of information. Here, we focus on the relevant data on learners, such as personal information devices, network address, and learner location and image. Our primary purpose collected factors and them data safety rather than focusing on one element for determing participant's learning.

In our system the client application is installed from Smartphone devices using the Android operating system that supports GPS, and built-in Camera, which allows one to capture learning objects. In particular, we use many solutions such as image capture, which is stored in memory variables instead of from files and data are transmitted from the client to the Virtual Learning Environment data (VLE data), which is encrypted.

The contribution of this paper is that it outlines a secure solution, as well as variable management and image size. In addition, it detailes the combination of information related to making learning object assessment more accurate. With data storage systems that we built on the system from the Virtual Learning Environment (VLE) by Moodle software and PHP server on website "http://mobile.ona.vn," teachers and learners are allowed to review their information, communicate with each other on the web server and restrictions and directions for development are gathered.

We chose the Android platform (version 2.3) of the Google and Virtual Learning Environment (MLE) on the PHP server, as it requires one to build 1 server API (Application Programming Interface) which allows communication between the Android operating system and the PHP web server. Our evaluations show that the use of GPS to locate the object is more efficient than the use of IP. Additionally, our solution ensures safety from the client application when image learning objects are taken directly from the camera instead of the image file and the image data coding (encoding Base64) and confirmation of the password encryption (one-way MD5 encryption with salt) is transmitted to the server API for decoding and comparing with the stored data storage is secure.

The rest of the paper is organized as follows:

In section 2, we introduce our client system platform built on Android mobile devices. In the third section, we present the Virtual Learning Environment system built on the PHP web server including a database structure that we use to store data from the Android client- a server API enables communication between two systems. In the fourth section, we present a security analysis to use multiple methods of identifing objects from the client and the server. In the fifth section, we present some implementations from our system on telephone equipment and some evaluation 
regarding image size and time when its data is transmitted to our system. Finally, we present conclusions and future work.

\section{ANDROID CLIENT}

Our client system is built on a Android mobile platform device. Figure 1 shows the workflow of AVLI when it is executed on a mobile device.

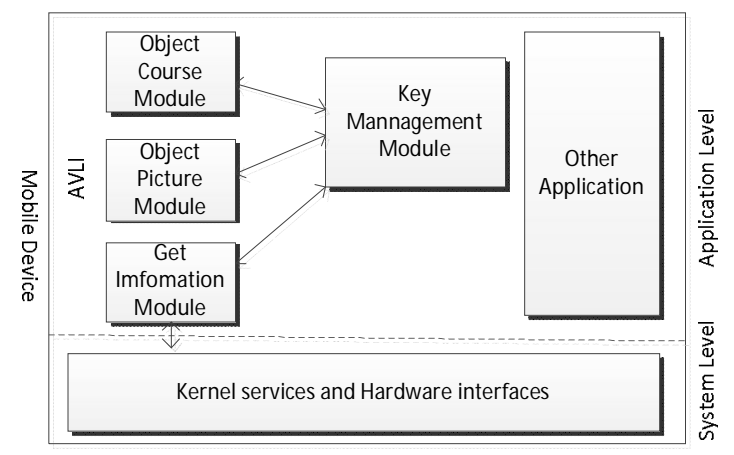

Figure 1. AVLI Client runs on Smartphone

The AVLI system includes three modules: the first module certification object participated (based on user and password), and the second module to acquire images directly from the camera. Both modules are encrypted, including the first module which is encrypted using the MD5 encryption method (one-way encryption) combined with the salt [4], the second module is encrypted with the Base64 method. The third module gathers other information to clarify the object, as follows:

\subsection{Login Object Course Module:(Figure 2)}

Course Database is stored in table "user" and includes "user" and "password" information. This module is implemented by the client to input "user" and "password." After that, the password will be encrypted (MD5 encrypted + salt) before it is sent from the client to the server API to compare with values stored in the table "user" on the VLE system.

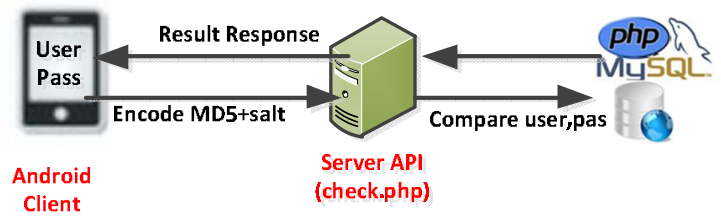

Figure 2. Object Course Checking

We use the MD5 hash function of the Java language to establish a one-way encryption to provide the security of password and combine "salt" to increase difficulty of cracking. This solution is synchronized from the client and server. 


\subsection{Object Image module:}

The teacher or education manager of the Virtual Learning Environment System Database (VLE Data) create the properties that the AVLI system would like to store, such as: picture, GPS (Latitude, Longitude), network (IP and MAC), Config of mobile: IMEI. Its structure is described in Figure 3:

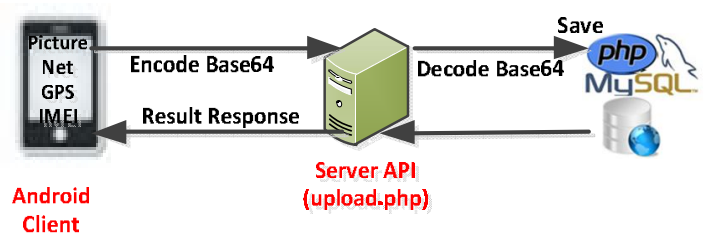

Figure 3. Object Picture module

\subsection{Image Encryption Method:}

Base64 is a group of similar binary-to-text encoding schemes that represent binary data in an ASCII string format by translating it into a radix-64 representation. Base64 is commonly used in a number of applications including email via MIME, and storing complex data in XML [2].

We use the Base64 method to encode and decode. Base64 class is held as follows (see Figure 4):

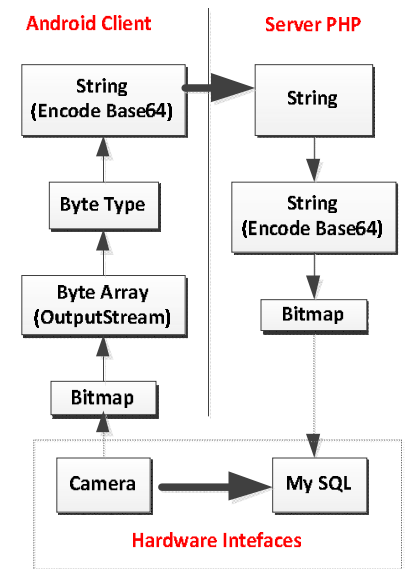

Figure 4. Upload picture from Android to Server PHP

\subsection{Get Information module:}

This module will take the MAC address, IP address of the device access and configuration devices "IMEI" (International Mobile Station Equipment Identity). The IMEI number is used by a GSM network to identify valid devices and therefore can be used for stopping a stolen phone from accessing that network, it alos includes information on the origin, model, and serial number of the device [3]. 


\section{VIRTUAL LEARNING ENVIRONMENT}

Moodle is a Course Management System (CMS), also known as a Learning Management System (LMS) or a Virtual Learning Environment (VLE) [5]. It is a free web application that educators can use to create effective online learning sites. We have built a Host and Domain "mobile.ona.vn." It allows us to create online learning environments with configurations as follows: model 1.9 (software), PHP 4.3.0 (language), and MySQL 4.1.16 (database). In which, we build a course "Android Virtual Learning Identification" with a Virtual Learning Environment System Database (VLE Data) and "Proof and identify learners Database" to store the value obtained from AVLI system.

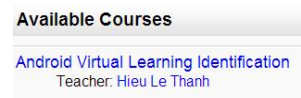

Teacher: Hieu Le Thanh
A Privacy - Identfication and Learning objects tracking System for Smartphones based on Environment Database

Figure 5. A course on mobile.ona.vn

\subsection{AVLI system:}

\subsubsection{Structure (Figure 6):}

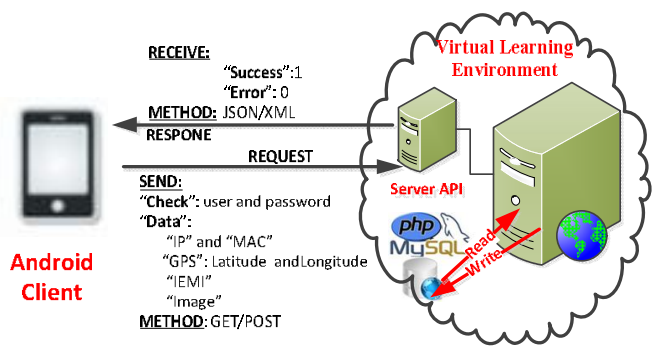

Figure 6. Communication between Client and Virtual Learning Environment Database

3.1.2. The server API module is written in PHP language in our system on "mobile.ona.vn" website. It allows for the receiving of encrypted string data to decrypt and to be stored in an mysql database or directory. The algorithm is as follows:

a. Server API access mysql database:

POST (data); //gets the data from client

Mysql_select_db(database); //access database

Mysql_query(table); // query command

-Update (field); //saves data from client to database on server

-Select (field); //find data

-Get filed send to client

b. Server API saves an image from the camera

POST (data); //gets the data from client

Mysql_select_db(database); //access database

Mysq1_query(table); // query command

-Select (field); //find a folder to save the image

-Save the file: fopen(), fwrite(), fclose(); 
International Journal of Computer Science \& Information Technology (IJCSIT) Vol 5, No 5, October 2013

\subsubsection{Virtual Learning Environment Database Structure (my SQL):review figure 7}

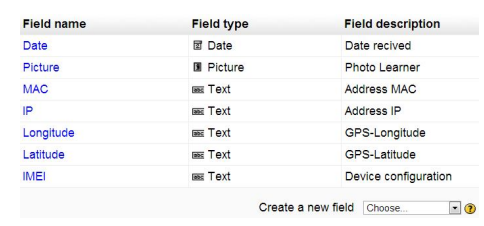

Figure 7. Virtual Learning Environment Database Structure

\section{SECURITY ANALYSIS}

4.1. Take an image from the camera: take the photo from an image file or use image variables. (see Table 1):

Table 1

Compare camera pictures taken from file or from memory variables

\begin{tabular}{|l|l|l|}
\hline Issue & File & Variables \\
\hline $\begin{array}{l}\text { Customiz } \\
\text { Speed }\end{array}$ & Easy review or editing & No editing \\
\hline Memory & $\begin{array}{l}\text { Can store multiple images and } \\
\text { variable space is much smaller } \\
\text { than the size of the extend } \\
\text { memory }\end{array}$ & $\begin{array}{l}\text { The number of images stored depends on } \\
\text { the limit of internal memory }\end{array}$ \\
\hline $\begin{array}{l}\text { File } \\
\text { space }\end{array}$ & $\begin{array}{l}\text { It can compress the image by } \\
\text { variables or compress software }\end{array}$ & It can compress images to a smaller size \\
\hline
\end{tabular}

The communication between the phone and the network system need latency time.

4.2. Confirm location of learning object by GPS: a comparison with the use IP. (see Table 2):

Table 2

Compare location of objects between GPS and IP

\begin{tabular}{|l|l|l|}
\hline No. & GPS & IP \\
\hline $\begin{array}{l}\text { Connectio } \\
\text { n devices }\end{array}$ & $\begin{array}{l}\text { One: Smartphone } \\
\text { must have GPS }\end{array}$ & $\begin{array}{l}\text { Many: network card or wifi or USB } \\
\text { 3G or GPRS } \\
\text { (Via cellular networks) }\end{array}$ \\
\hline Speed & $\begin{array}{l}\text { Depending on the } \\
\text { signal and weather }\end{array}$ & $\begin{array}{l}\text { MB number can download } \\
\text { information from mobile subscribers }\end{array}$ \\
\hline Support & $\begin{array}{l}\text { Google Maps API, } \\
\text { map Baidu API... }\end{array}$ & $\begin{array}{l}\text { Difficult to determine based on the IP } \\
\text { address when accessing the network }\end{array}$ \\
\hline Accuracy & High & $\begin{array}{l}\text { Low or undefined if the IP address is } \\
\text { changed using virtual IP, VPN, }\end{array}$ \\
\hline
\end{tabular}


4.3. Security evaluation of encryption methods includes: encode MD5+salt and encode Base64.

4.3.1. Encode password by "MD5+salt" method: this is a one-way encryption, it allows for encryption but not decryption. Thus, when only the password is raised, the new password is changed instead of bowing to find the password. In addition, "Salt" combined with MD5 encryption makes the system more secure[4].

4.3.2. Encode by the Base 64 bit method: Base 64 encoding schemes are commonly used when there is a need to encode binary data that needs to be stored and transferred over media that are designed to deal with textual data. It is suitable for using encryption to image and text data such as GPS, IP, and MAC in the AVLI system.

\section{IMPLEMENTATION AND EVALUATION}

As Android is the most popular mobile OS, we implement a prototype of AVLI on Lenovo P70, supported by Android 2.3 Gingerbread, 512MB of RAM. The system hosting the experiments was host domain: mobile.ona.vn and it was installed on Moodle software. It was connected to the Internet with wifi. We programmed AVLI in java using Eclipse IDE with an Android SDK, developed the software on an Android emulator and ran it on a real phone. Figure 8 shows the modules of the AVLI implementation.

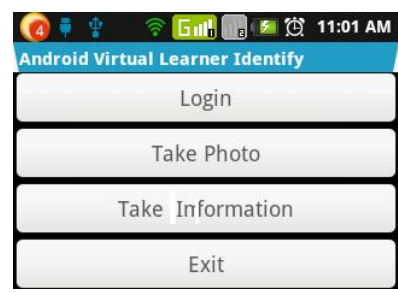

Figure 8. Android Virtual Learner Identify System

We obtain location data from Lenovo P70 with values: longitude: 114.40672, latitude: 30.517261666666666 and called upon Google Maps to display the point of the learner in maps (Figure 9). In the future, we will embed Google Maps API in the Android system to track learners and their coordinates for more intuition $[15,16,17]$.

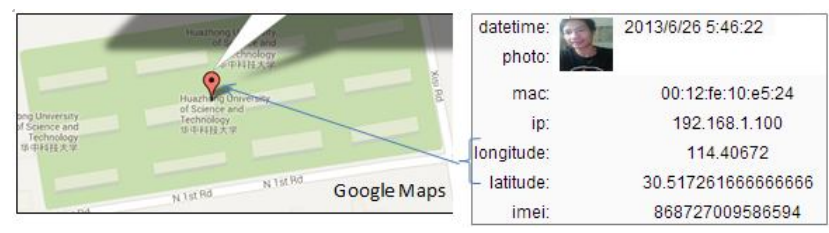

Figure 9. Virtual Learning Environment Database Structure

Device configuration information such as: time, date and IP address that learners are able to access via the school's network or not during school hours or outside school hours. In addition, some software uses GPS and IMEI which not only allows one to identify the owner of the phone device being accessed, or check some information about the device [6], but also allows one to search the missing device [7]. 
International Journal of Computer Science \& Information Technology (IJCSIT) Vol 5, No 5, October 2013

An image is saved as a folder with its structure as a number. That number is a stored value corresponding with table.ID in the database. Figure 10, folder: mobile.ona.vn/data/4/moddata/9/22/15 is the position of an image [10]: mdl_course.id=4 (No. course), mdl_data.id=9 (name database), mdl_data_fields.id=22 (No. image field) and mdl_data_records.id=15 (No. user). We use PHPMyAdmin to review database structure.[11]

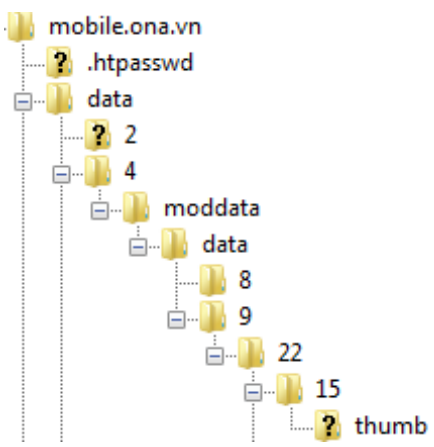

Figure 10. Folder Structure to save the image

For effectiveness of the compressed image, data transmitted from the AVLI system before the image is uploaded onto the Database's Virtual Learning Environment (VLE Data), we use System.currentTimeMillis(); [8] function to get the time and bitmap.compress (Bitmap.CompressFormat.PNG,\% compression, stream); [9] function to select the image compression rate, the following results in table 3 :

Table 3

Compare the image compression rate with time to upload them

\begin{tabular}{|c|c|}
\hline \% Compression & Time (milliseconds) \\
\hline 100 & 1143 \\
\hline 90 & 1103 \\
\hline 80 & 1089 \\
\hline 70 & 1076 \\
\hline 60 & 1057 \\
\hline 50 & 1034 \\
\hline 40 & 1021 \\
\hline 30 & 1012 \\
\hline 20 & 1007 \\
\hline 10 & 965 \\
\hline
\end{tabular}

From Table 3, we found that with the reduced compression rate, the image size will be smaller and the transmission time will be faster. However, image quality is inversely proportional to the reduced compression ratio and the quality of images captured is also determined by the camera. We chose a compression rate of $90 \%$ for image upload and review; the system still recognized the faces of the learner object.

The source code of AVLI system includes: 3983 lines java, 443 lines xml code, 100 lines PHP code and the installation package size on an Android is $69 \mathrm{~KB}$. 


\section{RELATED WORK}

The Smartphone is a unique device with the following devices rolled into one: phone, PC, networked device, audio/video capable of recording and playback, GPS enabled, live TV, gaming device, accelerometer. Inputs to mobile could be through many interfaces including: keypad, touch screen, camera (photo \& video), barcode scanners, microphone voice, motion sensor, and network input to GPS [12].

These features are integrated more on the computer, allowing learners to easily access course content, as well as permission to use these functions to define learning objects which outperformed the computer. But the issues with them are higher development cost and no cross platform support. Thus, the learning application is tied down to one platform[13].

During the implementation process of our system, we had to install the application MLE (Mobile Learning Engine) and Mono (Mobile Moodle) in website mobile.ona.vn. This project was not supported in later versions of the Android and Java version and was configuration dependent. In addition, support the development of client applications, but do not take advantage of the existing infrastructure of the course's teaching Virtualization Environment.

The organisational process we teach in this system also faced difficulties in identifying and selecting a teaching object mobile platform after deciding on the Android platform as they are widely used to solve the issue of the costs of Smart phones.

The integrity and identification of online learning also been mentioned in [14], : Ninety-six percent $(96 \%)$ of the respondents indicate their college requires learners to authenticate their identity — using a unique username and password — to access their online course and user.id in distance education are not secure. They take place in circumstances that include firm learner identification such as: time limits, and sending emails to confirm receipt.

\section{Conclusions}

We have built a virtual learning environment system (domain mobile.ona.cn) including applications for mobile devices (AVLI system). The system uses the database to help teachers and education managers to identify learning objects by collecting information about the object properties. Such information includes: user location, IP address, image and time; the inclusion of such elements would increase accuracy of user authentication.

Our proposed approach to safety in the process of communication between the phone devices and the server, especially between the Android system and the PHP server is to use the password encryption solution, and encryption images.

We present the design, implementation and security analysis and evaluation of the AVLI system. Finally, we present the problem in the future development and management of our system.

\section{REFERENCES}

[1] Gartner, (07/2013) "Says Asia/Pacific Led Worldwide Mobile Phone Sales to Growth in First Quarter of 2013".

[2] Wikipedia, (2013) "Base64", https://en.wikipedia.org/wiki/Base64. 
International Journal of Computer Science \& Information Technology (IJCSIT) Vol 5, No 5, October 2013

[3] Wikipedia, (2013) "International Mobile Station Equipment Identity (IMEI)", http://en.wikipedia.org/wiki/International_Mobile_Station_Equipment_Identity.

[4] Viral Patel, (06/08/2012) "Java MD5 Hashing \& Salting: Secure Your Passwords", http://viralpatel.net/blogs/java-md5-hashing-salting-password/.

[5] Moodle, (2013) https://moodle.org/?lang=vi,.

[6] Website checks regarding information about the device: (07/2013) "IMEI" from http://www.imei.info/, http://www.numberingplans.com/.

[7] ZhaoKao, JinHai, DeQingZou,WangDaiBin, , (2012) "A Privacy-Preserving Location Tracking System for Smartphones Based on Cloud Storage", Mobicom13, p.234.

[8] (07/2013) "AndroidAPIs,java.lang.System », http://developer.android.com/reference /java/lang/System.html\#currentTimeMillis(), 07/2013.

[9] AndroidAPIs, (07/2013) “ android.graphics.Bitmap », http://developer.android.com/ reference/android/graphics/Bitmap.html\#compress(android.graphics.Bitmap.CompressFormat, int, java.io. OutputStream), 2013.

[10] Sau Hi, (07/2013) "Learn how to use FTP by FileZilla", www.youtube.com/watch?v=G9wCcq4bAgU

[11] ituplusdotcom, (07/2013) "Learn how to use PHPMyAdmin", www.youtube.com/watch?v=J4PoclzoO8

[12] Amit Garg, (07/2013) "How To Create Successful M-Learning Strategy", http://www.upsidelearning.com/blog/index.php/2010/06/23/how-to-create-successful-m-learningstrategy-mlearncon-part-i/.

[13] Amar Jadhav, (03/2010) "Tools For Mobile Learning Development", http://www.upsidelearning.com/blog/index.php/2010/03/21/tools-for-mobile-learning-development/.

[14] Mike Jortberg, (07/2013) "Mike.Jortberg@ Acxiom.com, "Academic Integrity and Identity in Online Learning", Midwest Blackboard User's Group (SLATE) Meeting at Lewis University.

[15] Wikipedia, (07/2013) “Longitude”, http://en.wikipedia.org/wiki/Longitude, 07/2013.

[16] Wikipedia, (07/2013) "Latitude", http://en.wikipedia.org/wiki/Latitude,.

[17] (07/2013) "How To Convert a Decimal to Sexagesimal", http://geography.about.com/library/howto/htdegrees.htm.

\section{Authors}

Le Thanh Hieu was born in Hue city, VietNam, in 1976. He received the Diploma in Informatics from Hue University's college of Education and became a lecturer in there from 1998. He also holds a Master's of Computer science in School Information and Communication Technology of the HaNoi University of Science and Technology. Currently, Dr. Le Thanh Hieu is Ph.D student of Computer Science Department, HuaZhong University of Science and Technology, Wuhan, China. His research interests are in the fields of elearning and education management security and privacy.

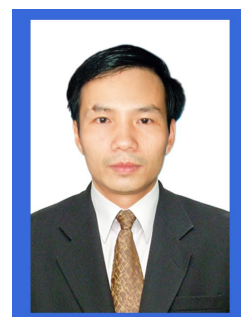

